\title{
COMPARACIÓN ENTRE DOS MÉTODOS DE VENTILACIÓN EN LA COMPOSICIÓN QUÍMICA DE COMPOST DE ESTIÉRCOLES PECUARIOS
}

\author{
Sonia LÓPEZ FERNÁNDEZ ${ }^{1}$, Rodolfo SERRATO CUEVAS ${ }^{2}$, \\ Octavio Alonso CASTELÁN ORTEGA ${ }^{3}$ y Francisca AVILÉS NOVA ${ }^{1 *}$
}

${ }^{1}$ Centro Universitario Temascaltepec, Universidad Autónoma del Estado de México, km. 67.5 carretera TolucaTejupilco, Barrio de Santiago, 51300 Temascaltepec de González, Estado de México, México

${ }^{2}$ Facultad de Ciencias Agrícolas, Universidad Autónoma del Estado de México, El Cerrillo Piedras Blancas, 50090 Toluca, Estado de México, México

${ }^{3}$ Facultad de Medicina Veterinaria y Zootecnia, Universidad Autónoma del Estado de México, El Cerrillo Piedras Blancas, 50090 Toluca, Estado de México, México

*Author for correspondence; franavilesnova@yahoo.com.mx

(Recibido marzo 2016; aceptado agosto 2017)

Palabras clave: contenido nutrimental, residuos orgánicos, aireación, compostaje

\section{RESUMEN}

Este trabajo comparó el efecto de dos métodos de ventilación en la composición química de cuatro compost de estiércoles pecuarios. Para cada tipo de estiércol mezclado se realizó un experimento de compostaje. En cada experimento (Exp) se ensayaron dos métodos de ventilación: manual con pala $(\mathrm{Vm})$ y con tubos de policloruro de vinilo perforados (Vt). El Exp 1 se denominó compost estiércol bovino (CBo + Vm, CBo $+\mathrm{Vt}$ ), el Exp 2 compost estiércol caprino $(\mathrm{CCa}+\mathrm{Vm}, \mathrm{CCa}+\mathrm{Vt})$, el Exp 3 compost estiércol equino $(\mathrm{CEq}+\mathrm{Vm}, \mathrm{CEq}+\mathrm{Vt})$ y el Exp 4 compost estiércol ovino $(\mathrm{COv}+\mathrm{Vm}$, $\mathrm{COv}+\mathrm{Vt})$. Durante el proceso se analizaron temperatura, potencial de hidrogeno $(\mathrm{pH})$, conductividad eléctrica $(\mathrm{CE})$, materia orgánica $(\mathrm{MO})$, carbono orgánico $(\mathrm{C})$, nitrógeno total $(\mathrm{N})$, relación carbono/nitrógeno $(\mathrm{C} / \mathrm{N})$, fosfato $\left(\mathrm{PO}_{4}\right)$, potasio $(\mathrm{K})$, magnesio $(\mathrm{Mg})$, sulfato $\left(\mathrm{SO}_{4}\right)$, calcio $(\mathrm{Ca})$, sodio $(\mathrm{Na})$, hierro $(\mathrm{Fe})$, manganeso $(\mathrm{Mn})$, boro $(\mathrm{B})$, zinc $(\mathrm{Zn})$ y cobre $(\mathrm{Cu})$. Para cada experimento se utilizó un diseño completamente aleatorizado con tres repeticiones. En las variables de composición química se realizó un análisis de varianza de un factor utilizando el programa Minitab y las medias se compararon con la prueba de Tukey $(\mathrm{p}<0.05)$. La temperatura en cada experimento mostró diferencias significativas entre tratamientos $(\mathrm{p}=0.000)$. Los productos provenientes de $\mathrm{CBo}, \mathrm{CCa}$ y COv no presentaron diferencias en la composición química por el método de ventilación $(\mathrm{p}>0.05)$. El producto proveniente de $\mathrm{CEq}+\mathrm{Vt}$ presentó menor relación $\mathrm{C} / \mathrm{N}$ $(\mathrm{p}=0.049)$. Los métodos de ventilación no influyeron en las características químicas de los compost de estiércol bovino, caprino y ovino, sin embargo, en el compost de estiércol equino al colocar tubos en las pilas de compostaje, se favoreció la degradación de la $\mathrm{MO}$ y redujo la relación $\mathrm{C} / \mathrm{N}$. Se concluye que los dos métodos de ventilación se recomiendan en los $\mathrm{CBo}, \mathrm{CCa}$ y $\mathrm{COv}$. Y el método de ventilación con tubos se recomienda en el CEq.

Key words: nutrient content, organic waste, aeration, composting 


\begin{abstract}
In this work we compared the effects of two ventilation methods on the chemical composition of four livestock manure compost. We conducted four manure composting experiments (Exp) with two ventilation methods: manually with a shovel (Vm), and with perforated polyvinyl chloride $(\mathrm{Vt})$. Exp 1 was designated cattle manure compost $(\mathrm{CBo}+\mathrm{Vm}, \mathrm{CBo}+\mathrm{Vt})$; Exp 2, goat manure compost $(\mathrm{CCa}+\mathrm{Vm}, \mathrm{CCa}+\mathrm{Vt})$; Exp 3 , horse manure compost $(\mathrm{CEq}+\mathrm{Vm}, \mathrm{CEq}+\mathrm{Vt})$, and Exp 4, sheep manure compost $(\mathrm{COv}+\mathrm{Vm}, \mathrm{COv}+\mathrm{Vt})$. A completely randomized design was used in each experiment, with three repetitions. A one-way analysis of variance was conducted using the Minitab program and Tukey's test $(\mathrm{p}<0.05)$ to compare methods. We analyzed temperature, hydrogen potential $(\mathrm{pH})$, electric conductivity $(\mathrm{EC})$, organic matter $(\mathrm{OM})$, organic carbon $(\mathrm{C})$, total nitrogen $(\mathrm{N})$, carbon/nitrogen ratio $(\mathrm{C} / \mathrm{N})$, phosphate $\left(\mathrm{PO}_{4}\right)$, potassium $(\mathrm{K})$, magnesium $(\mathrm{Mg})$, sulfate $\left(\mathrm{SO}_{4}\right)$, calcium $(\mathrm{Ca})$, sodium $(\mathrm{Na})$, iron $(\mathrm{Fe})$, manganese $(\mathrm{Mn})$, boron $(\mathrm{B})$, zinc $(\mathrm{Zn})$ and copper $(\mathrm{Cu})$. There were highly significant differences in the temperatures observed with the different treatments in the experiments $(\mathrm{p}=$ 0.000). No statistical differences were observed in the chemical composition in CBo, $\mathrm{CCa}$ and $\mathrm{COv}$ based on the ventilation method used $(\mathrm{p}>0.05)$. Statistical differences in $\mathrm{C} / \mathrm{N}$ were found in $\mathrm{CEq}+\mathrm{Vt}(\mathrm{p}=0.049)$. The ventilation methods did not impact the chemical characteristics of the cattle, goat and sheep manure composts, but in CEq it was found that placing plastic tubes in the compost piles facilitated greater degradation of the $\mathrm{OM}$ and reduced the $\mathrm{C} / \mathrm{N}$. Both ventilation methods are recommended in $\mathrm{CBo}, \mathrm{CCa}$ and $\mathrm{COv}$. And the ventilation method with tubes is recommended in $\mathrm{CEq}$.
\end{abstract}

\section{INTRODUCCIÓN}

La intensificación de la producción ganadera aumenta la generación de estiércol, lo cual origina una gran cantidad de nutrientes desechados que se concentran en áreas específicas dentro de la unidad productiva (Pinos et al. 2012). Esto puede causar graves problemas ambientales como translocación de microorganismos patógenos y semillas de maleza, sales inorgánicas e incluso trazas de metales pesados (Lazcano et al. 2008). Igualmente, pueden causar un impacto negativo en el ambiente como malos olores, emisiones de gases y contaminación de suelo y agua (Bernal et al. 2009). En México, la regulación y vigilancia gubernamental sobre el uso y manejo del estiércol animal es escasa y confusa, y carece de especificaciones claras (Pinos et al. 2012). Esto ha propiciado un abuso en las descargas de desechos en cuerpos de agua y suelos de cultivo. El compostaje es el método más adecuado en el tratamiento eficaz de desechos orgánicos para obtener un producto higiénicamente seguro y económicamente rentable (Kulcu y Yaldiz 2007, Szabová et al. 2010). El nitrógeno (N) disponible para las plantas a partir de estiércoles es altamente dependiente de la composición de los mismos ( $\mathrm{Li}$ y Li 2014), y la calidad del estiércol varía en relación con el tipo de animal, su edad, dieta y sistema de manejo (Chadwick et al. 2000).
Azzez y van Averbeke (2010) mencionaron que las relaciones carbono/nitrógeno $(\mathrm{C} / \mathrm{N})$, carbono/fósforo $(\mathrm{C} / \mathrm{P})$ y nitrógeno/fósforo $(\mathrm{N} / \mathrm{P})$ en los estiércoles de diferentes especies animales son reflejo de diferentes prácticas de manejo, patrones de alimentación de los animales y composición de los forrajes en el caso de los rumiantes. La conservación de nutrientes en el producto final de un proceso de compostaje es importante para no afectar su valor agronómico y su potencial como abono orgánico

Los factores involucrados en el proceso de compostaje como aireación, relación inicial $\mathrm{C} / \mathrm{N}$ y contenido de humedad influyen en el contenido de nutrientes, madurez y estabilidad del compost (Guo et al. 2012). La excesiva aireación puede incrementar pérdidas de amoniaco $\left(\mathrm{NH}_{3}\right)$ y conducir a un proceso más lento; una relación inicial $\mathrm{C} / \mathrm{N}$ baja puede incrementar pérdidas de $\mathrm{N}$ como gas amoniaco y el contenido de humedad puede afectar la calidad del compost aunque no significativamente (Guo et al. 2012). Parkinson et al. (2004) sugirieron reducir los volteos de tres a uno a las pilas de compost durante todo el proceso, para reducir las pérdidas de nutrientes con estiércol bovino. El objetivo de este trabajo fue comparar dos métodos de ventilación, uno manual con pala y otro con tubos perforados de policloruro de vinilo, en la composición química de cuatro compost elaborados con estiércol pecuario. 


\section{MATERIALES Y MÉTODOS}

El proceso de compostaje se realizó de noviembre de 2014 a enero de 2015 en el Rancho "El Salitre" del Centro Universitario UAEM-Temascaltepec, ubicado en el municipio de San Simón de Guerrero en la región subtropical del sureste del Estado de México, a $100^{\circ} 6^{\prime}$ $27^{\prime \prime} \mathrm{O}, 19^{\circ} 2^{\prime} 8^{\prime \prime} \mathrm{N}$ y $1800 \mathrm{msnm}$. El clima es templado subhúmedo con lluvias en verano (Cw) (INEGI 2009). Los sustratos orgánicos utilizados para la elaboración de los compost fueron estiércoles bovino, caprino y ovino mezclados con residuos de paja de maíz, y estiércol equino mezclado con residuos de paja de avena. Los estiércoles se recolectaron en el piso de los corrales techados, donde se habían acumulado por dos meses. La alimentación diaria de los bovinos fue forraje verde de maíz a libre acceso y $2 \mathrm{~kg}$ de suplemento alimenticio a base de soya, maíz molido, sorgo y sales minerales (MULTISAL; contenido en 1000 g: Cu, 600 mg; Fe, 1920 mg; Mn, 2066.46 mg; Co, 6 mg; I 19.84, mg; Zn, 3000.24 mg; Se, 12 mg; P, 59220 mg; Mg, 2000.32 mg; Ca, 281 mg; Na, 703.20 $\mathrm{mg}, \mathrm{y} \mathrm{K}, 2439 \mathrm{mg}$ ). La alimentación de los caprinos y los ovinos se basó en pastoreo diario en gramíneas y arbustos nativos, además de un suplemento de 250 $\mathrm{g}$ /día/animal a base de rastrojo de maíz, sorgo molido, maíz molido, pasta de soya y sales minerales (MULTISAL). La alimentación de los equinos consistió en avena henificada y agua a libre acceso.

En cada tipo de estiércol mezclado se realizó un experimento de compostaje utilizando dos métodos de ventilación: uno manual con pala $(\mathrm{Vm})$, y otro con tubos perforados de policloruro de vinilo $(\mathrm{Vt})$. En cada experimento (Exp.) se utilizó un diseño completamente aleatorizado. Los tratamientos fueron los siguientes: Exp. 1: compost estiércol bovino (CBo $+\mathrm{Vm}, \mathrm{CBo}$ $+\mathrm{Vt})$; Exp. 2: compost estiércol caprino $(\mathrm{CCa}+\mathrm{Vm}$, $\mathrm{CCa}+\mathrm{Vt})$; Exp. 3: compost estiércol equino $(\mathrm{CEq}+$ $\mathrm{Vm}, \mathrm{CEq}+\mathrm{Vt}$ ) y Exp. 4: compost estiércol ovino (COv $+\mathrm{Vm}, \mathrm{COv}+\mathrm{Vt}$ ), cada experimento tuvo tres repeticiones (pilas). La $\mathrm{Vm}$ consistió en voltear las pilas los días 15 y 30 posteriores al inicio del compostaje (El Kader et al. 2007) con una pala de excavación para jardín Trupper $(33.5 \times 26.2 \times 103 \mathrm{~cm})$. La Vt consistió en colocar horizontalmente dos tubos de policloruro de vinilo de $1 \mathrm{~m}$ de largo y $10 \mathrm{~cm}$ de diámetro en el interior de cada pila. A cada tubo se le realizaron tres perforaciones de $10 \mathrm{~cm}$ de diámetro separadas $15 \mathrm{~cm}$ entre sí, utilizando para ello un cuchillo caliente de acero. En cada extremo del tubo se dejó un margen de $20 \mathrm{~cm}$. Antes de formar las pilas de sustrato mezclado en el experimento, se tomaron al azar dos muestras de $1 \mathrm{~kg}$, las cuales se colocaron en bolsas de nylon previamente etiquetadas, se depositaron en cajas térmicas con bolsas de gel congeladas y se enviaron inmediatamente al laboratorio para su análisis químico. El cuadro I muestra el análisis químico de los estiércoles mezclados. En cada experimento los estiércoles se pesaron y humedecieron a un $60 \%$ con agua potable (analizador de humedad para suelo Keyway), excepto el estiércol bovino que contenía humedad mayor a $80 \%$; posteriormente se formaron seis pilas cónicas. Las pilas de los Exps. 1 y 4 pesaron $100 \mathrm{~kg}$ y midieron $0.8 \mathrm{~m}$ de alto $\times 1 \mathrm{~m}$ de diámetro en la base. En el Exp. 2 las pilas tuvieron un peso de $50 \mathrm{~kg}$ y sus medidas fueron de $0.6 \mathrm{~m}$ de alto $\times 0.8 \mathrm{~m}$ de diámetro en la base. En el Exp. 3 se formaron pilas de $50 \mathrm{~kg}$ con las mismas medidas que los Exps. 1 y 4, debido a la menor densidad del estiércol equino (báscula línea industrial, Nuevo León). En todos los experimentos las pilas se acomodaron al aire libre bajo la sombra de árboles de pino (Pinus sp.) sobre una película plástica de nylon.

CUADRO I. ANÁLISIS QUÍMICO DE LOS ESTIÉRCOLES MEZCLADOS

\begin{tabular}{lcccc}
\hline Parámetro & $\begin{array}{c}\text { Estiércol } \\
\text { bovino }\end{array}$ & $\begin{array}{c}\text { Estiércol } \\
\text { caprino }\end{array}$ & $\begin{array}{c}\text { Estiércol } \\
\text { equino }\end{array}$ & $\begin{array}{c}\text { Estiércol } \\
\text { ovino }\end{array}$ \\
\hline $\mathrm{pH}$ & 8.14 & 8.32 & 8.01 & 7.99 \\
$\mathrm{CE}(\mathrm{dS} / \mathrm{m})$ & 3.52 & 4.79 & 4.48 & 5.39 \\
$\mathrm{MO}(\%)$ & 87.45 & 78.45 & 86.79 & 84.95 \\
$\mathrm{C}(\%)$ & 50.73 & 45.51 & 50.34 & 49.28 \\
$\mathrm{~N}(\%)$ & 2.04 & 2.05 & 1.21 & 2.19 \\
$\mathrm{C} / \mathrm{N}$ & 24.87 & 22.20 & 41.60 & 22.50 \\
$\mathrm{PO}(\mathrm{mg} / \mathrm{kg})$ & 6880 & 2560 & 10140 & 11050 \\
$\mathrm{~K}(\mathrm{mg} / \mathrm{kg})$ & 15800 & 18800 & 6500 & 18800 \\
$\mathrm{Mg}(\mathrm{mg} / \mathrm{kg})$ & 3000 & 5700 & 880 & 3800 \\
$\mathrm{SO}(\mathrm{mg} / \mathrm{kg})$ & 600 & 2940 & 5166.67 & 3333.33 \\
$\mathrm{Ca}(\mathrm{mg} / \mathrm{kg})$ & 13500 & 36200 & 2360 & 13900 \\
$\mathrm{Na}(\mathrm{mg} / \mathrm{kg})$ & 230 & 7300 & 790 & 2900 \\
$\mathrm{Fe}(\mathrm{mg} / \mathrm{kg})$ & 1090 & 3600 & 1150 & 1750 \\
$\mathrm{Mn}(\mathrm{mg} / \mathrm{kg})$ & 440 & 440 & 88 & 430 \\
$\mathrm{~B}(\mathrm{mg} / \mathrm{kg})$ & 1420 & 840 & 590 & 1460 \\
$\mathrm{Cu}(\mathrm{mg} / \mathrm{kg})$ & 13 & 30 & 1.2 & 15 \\
$\mathrm{Zn}(\mathrm{mg} / \mathrm{kg})$ & 150 & 360 & 24 & 155 \\
\hline
\end{tabular}

pH: potencial de hidrogeno; CE: conductividad eléctrica; MO: materia orgánica; $\mathrm{C}$ : carbono orgánico; N: nitrógeno total; $\mathrm{C} / \mathrm{N}$; relación carbono/nitrógeno; $\mathrm{PO}_{4}$ : fosfato, $\mathrm{K}$ : potasio; $\mathrm{Mg}$ : magnesio; $\mathrm{SO}_{4}$ : sulfato; $\mathrm{Ca}$ : calcio; Na: sodio; Fe: hierro; Mn: manganeso, B: boro; $\mathrm{Cu}$ : cobre, y $\mathrm{Zn}$ : zinc

Durante el proceso de compostaje, la humedad del sustrato se mantuvo al $60 \%$ en las pilas de los cuatro experimentos agregando agua potable. Asimismo, se midió la temperatura del sustrato en tres sitios seleccionados al azar dos veces por semana durante las primeras tres semanas y una vez en las 
restantes, utilizando un termómetro de carátula para compost TFA.

Posteriormente se tomaron al azar tres submuestras de cada pila a una profundidad de $10 \mathrm{~cm}$, obteniendo dos muestras compuestas de $1 \mathrm{~kg}$ por tratamiento (i.e., cuatro muestras/experimento), las cuales se manejaron de manera similar a las muestras de los estiércoles y se enviaron al laboratorio para su análisis. Las variables analizadas en los estiércoles y compost fueron: potencial de hidrogeno $(\mathrm{pH})$ por lectura potenciométrica en solución 1:5 de agua destilada/muestra (HANNA Instruments 8521); conductividad eléctrica (CE) $(\mathrm{dS} / \mathrm{m})$ con conductímetro DR-3900 PerkinElmer (relación 1:5); materia orgánica (MO) $(\%)$ por calcinación en mufla; carbono orgánico (C) (\%) mediante la ecuación $\mathrm{C}=\% \mathrm{MO} / 1.724$; nitrógeno total $(\mathrm{N})(\%)$ por el método MicroKjeldahl titulométrico; relación carbono/nitrógeno $(\mathrm{C} / \mathrm{N})$ por medio de la ecuación $\mathrm{C} / \mathrm{N}=\% \mathrm{C} / \% \mathrm{~N}$; fosfatos $\left(\mathrm{PO}_{4}\right)(\mathrm{mg} / \mathrm{kg})$ por el método Morgan colorimétrico; boro (B) $(\mathrm{mg} / \mathrm{kg})$ por el método Azomethine-H colorimétrico (espectrómetro ultravioleta/visible Cary 50); sulfatos $\left(\mathrm{SO}_{4}\right)(\mathrm{mg} / \mathrm{kg})$ por el método turbidimétrico; potasio $(\mathrm{K})$, magnesio $(\mathrm{Mg})$, sodio $(\mathrm{Na})$, calcio $(\mathrm{Ca})$, hierro $(\mathrm{Fe})$, zinc $(\mathrm{Zn})$, cobre $(\mathrm{Cu})$ y manganeso $(\mathrm{Mn})(\mathrm{mg} / \mathrm{kg})$ por absorción atómica LCH (digestión) (Analyst 400 Spectrometer PerkinElmer). Las características químicas de los estiércoles y los productos se analizaron según la norma mexicana NMX-FF-109-SCFI-2008 (SEECO 2008) en en laboratorio Phytomonitor por duplicado. Las normas mexicanas NTEA-006-SMA-RS-2006 (SMA 2006) y NADF-020-AMBT-2011 (SEDEMA 2012) se tomaron como referencia para comparar la calidad de los productos obtenidos.

Para cada experimento se utilizó un diseño completamente aleatorizado, con tres repeticiones por tratamiento. En todas las variables de composición química se realizó un análisis de varianza (ANDEVA) de un factor. La temperatura se analizó utilizando ANDEVA con medias repetidas a través del tiempo. Se usaron el comando del modelo general lineal (GLM, por sus siglas en inglés) del programa Minitab para Windows 12.21 y la comparación de medias a través de la prueba de Tukey $(\mathrm{p}<0.05)$.

\section{RESULTADOS Y DISCUSIÓN}

\section{Comportamiento de la temperatura durante el compostaje}

Los patrones de temperatura muestran la actividad microbianay la aparición de los procesos del compostaje
(Bernal et al. 2009). Según Insam y de Bertoldi (2007) la fase termófila ocurre cuando la temperatura del sustrato se encuentra entre 35 y $65^{\circ} \mathrm{C}$; cuando la temperatura varía de 30 a $45^{\circ} \mathrm{C}$ se presentan una alta tasa de biodegradación y la máxima diversidad microbiana. La figura 1 muestra la temperatura promedio de cada experimento durante el proceso de compostaje.

En los experimentos, la temperatura presentó diferencias altamente significativas entre los tratamientos $(\mathrm{p}=0.000)$, la edad del compost (días) $(\mathrm{p}=0.000)$ y la interacción $(\mathrm{p} \leq 0.01)$. En el Exp 1, el proceso $\mathrm{CBo}+\mathrm{Vt}$ presentó $2.0^{\circ} \mathrm{C}$ menos de temperatura que $\mathrm{CBo}+\mathrm{Vm}$, lo cual indica que los tubos perforados removieron el calor producido por la actividad microbiana. La temperatura en $\mathrm{CBo}+\mathrm{Vm}$ no se incrementó en los primeros 12 días, lo cual pudo deberse al exceso de humedad inicial del estiércol bovino. Sin embargo, se observó un incremento de temperatura después de los 13 días debido a la disminución de la humedad del sustrato, lo que fomentó la actividad microbiana; no obstante, no alcanzó la fase termófila. El exceso de humedad satura los poros e impide el intercambio gaseoso, por lo que puede conducir a un proceso anaeróbico (Diaz y Savage 2007). Durante el compostaje se evapora gran cantidad de agua y el contenido de humedad disminuye (Bernal et al. 2009). En el Exp 2, el proceso CCa + Vm presentó $3.8^{\circ} \mathrm{C}$ más de temperatura que $\mathrm{CCa}+\mathrm{Vt}$, los cuales iniciaron la fase termófila siete días después de iniciado el proceso (Fig. 1b), al alcanzar la mayor temperatura: $53.6{ }^{\circ} \mathrm{C}$ y $42.1{ }^{\circ} \mathrm{C}$, respectivamente. Las altas temperaturas durante el compostaje indican biodegradación activa de la materia orgánica (MO). Con el agotamiento gradual de esta última, la temperatura disminuye y la MO entra en la fase de curación (Luo et al. 2014). En este trabajo, la temperatura se consideró como uno de los indicadores finales del proceso; se estabilizó en $20^{\circ} \mathrm{C}$ a los 45 días, valor cercano a la temperatura media ambiental. También se consideraron la reducción de la relación $\mathrm{C} / \mathrm{N}$ y cambios perceptibles como textura, color y eliminación de mal olor (Mirabelli 2008). En el Exp 3, los tratamientos CEq + Vm y CEq + Vt no mostraron la fase termófila. El proceso $\mathrm{CEq}+\mathrm{Vm}$ alcanzó $3.8{ }^{\circ} \mathrm{C}$ más de temperatura que $\mathrm{CEq}+\mathrm{Vt}$. $\mathrm{En} \mathrm{CEq}+\mathrm{Vt}$ la temperatura fue decreciente durante todo el proceso (Fig. 1c). En el Exp 4, COv + Vm alcanzó mayor temperatura $\left(40.4^{\circ} \mathrm{C}\right)$ el día 13 , como resultado de una mayor actividad microbiológica, y posteriormente mostró una disminución gradual (Fig. 1d). Guo et al. (2012) reportaron que la tasa de aireación en el compostaje de heces de cerdo con paja 
(a)

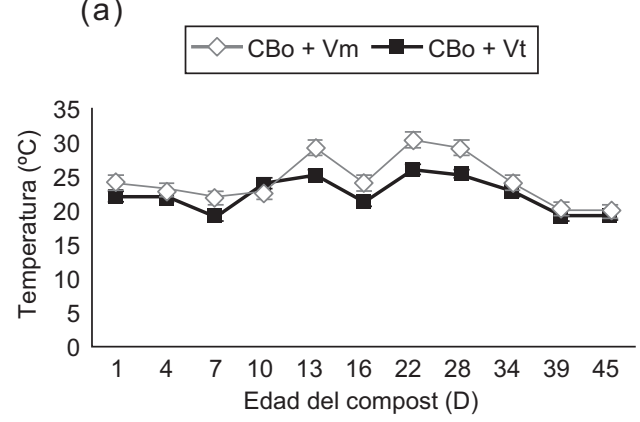

(c)

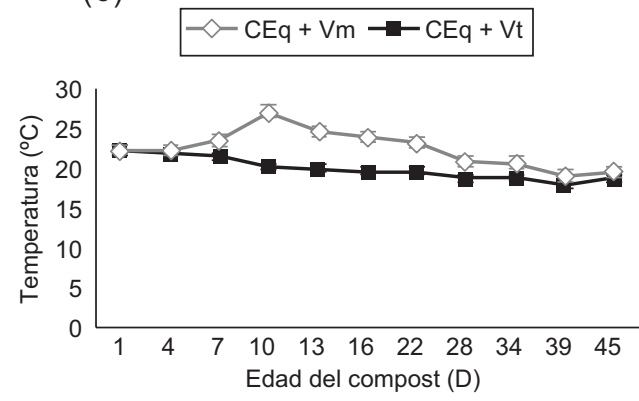

(b)

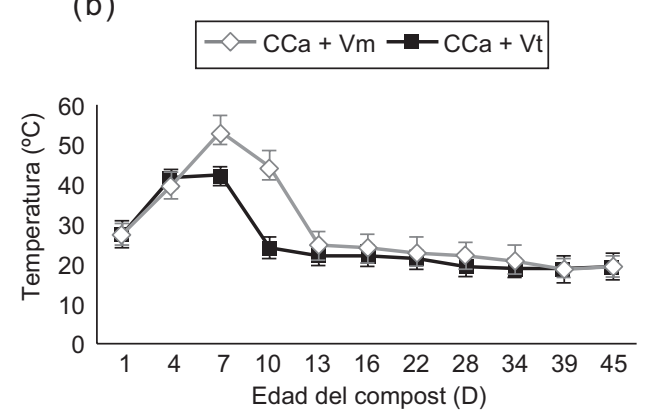

(d)

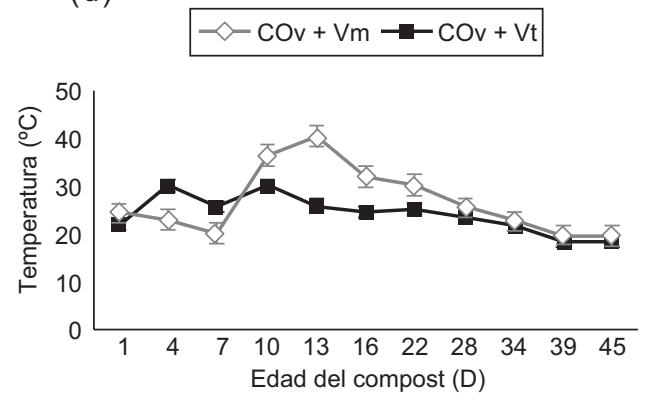

Fig. 1. Comportamiento de la temperatura durante los días (D) del proceso de compostaje en los experimentos: (a) $\mathrm{CBo}+\mathrm{Vm}$ (compost bovino con ventilación manual) y $\mathrm{CBo}+\mathrm{Vt}$ (compost bovino ventilado con tubos perforados); (b) $\mathrm{CCa}+\mathrm{Vm}$ (compost caprino con ventilación manual) y $\mathrm{CCa}+\mathrm{Vt}$ (compost caprino ventilado con tubos perforados); (c) $\mathrm{CEq}+\mathrm{Vm}$ (compost equino con ventilación manual) y CEq $+\mathrm{Vt}$ (compost equino ventilado con tubos perforados), y (d) $\mathrm{COv}+\mathrm{Vm}$ (compost ovino con ventilación manual) y COv $+\mathrm{Vt}$ (compost ovino ventilado con tubos perforados)

de maíz tuvo diferencias significativas en los cambios de temperatura $(\mathrm{p}=0.023)$. COv $+\mathrm{Vt}$ no alcanzó la fase termófila pero se observó actividad microbiológica por el incremento de temperatura durante los días cuatro y 10 (Fig. 1d). En los experimentos se observó que medir la temperatura solamente dos veces por semana durante las primeras semanas del compostaje limitó el análisis y pudo ser un factor para la falta de identificación de la fase termófila en los Exp 1 y Exp 3. En general los productos con Vt presentaron menor temperatura a través del tiempo, lo cual se atribuyó principalmente a la oxigenación constante del sustrato debido al efecto de los tubos perforados, los cuales removían el calor producido por la actividad microbiana.

\section{Caracterización de los productos del compostaje}

El cuadro II muestra las características químicas de los productos obtenidos en cada experimento.

Exp 1. El CBo no presentó diferencias significativas $(p>0.05)$ en cuanto a características químicas entre los métodos de ventilación. El pH presentó rangos alcalinos en los productos, valores que podrían deberse a la mayor liberación de bases relacionada con las sales minerales que recibían los animales en la dieta. La alcalinización en el compost es resultado de la producción amoniacal y la liberación de bases (Ruiz 2012). Brito et al. (2008) reportaron valores alcalinos en el $\mathrm{pH}$ (7.5 a 8.5) del compost de la fracción sólida de heces de ganado lechero, probablemente debido al efecto de amortiguamiento de los bicarbonatos. Oviedo et al. (2014) reportaron valores alcalinos en sus pilas de compostaje de biorresiduos de origen municipal debido principalmente al gran contenido de potasio. La CE del CBo aumentó respecto del contenido en el estiércol. Cáceres et al. (2006) reportaron aumento en la CE al final del proceso de compostaje de la fracción sólida de estiércol bovino; sin embargo, sus valores fueron menores ( 3 a $5 \mathrm{dS} / \mathrm{m}$ ) a los encontrados en este trabajo en $\mathrm{CBo}$, lo cual podría atribuirse al contenido de sales minerales en la suplementación que recibieron los animales y a la mineralización de la MO. Al avanzar el proceso de compostaje se incrementa la concentración de sales solubles, las cuales reflejan el progreso en la mineralización de la MO y contribuye al incremento de la salinidad (Cáceres et al. 2006). La norma NADF020-AMBT-2011 (SEDEMA 2012) establece el uso 


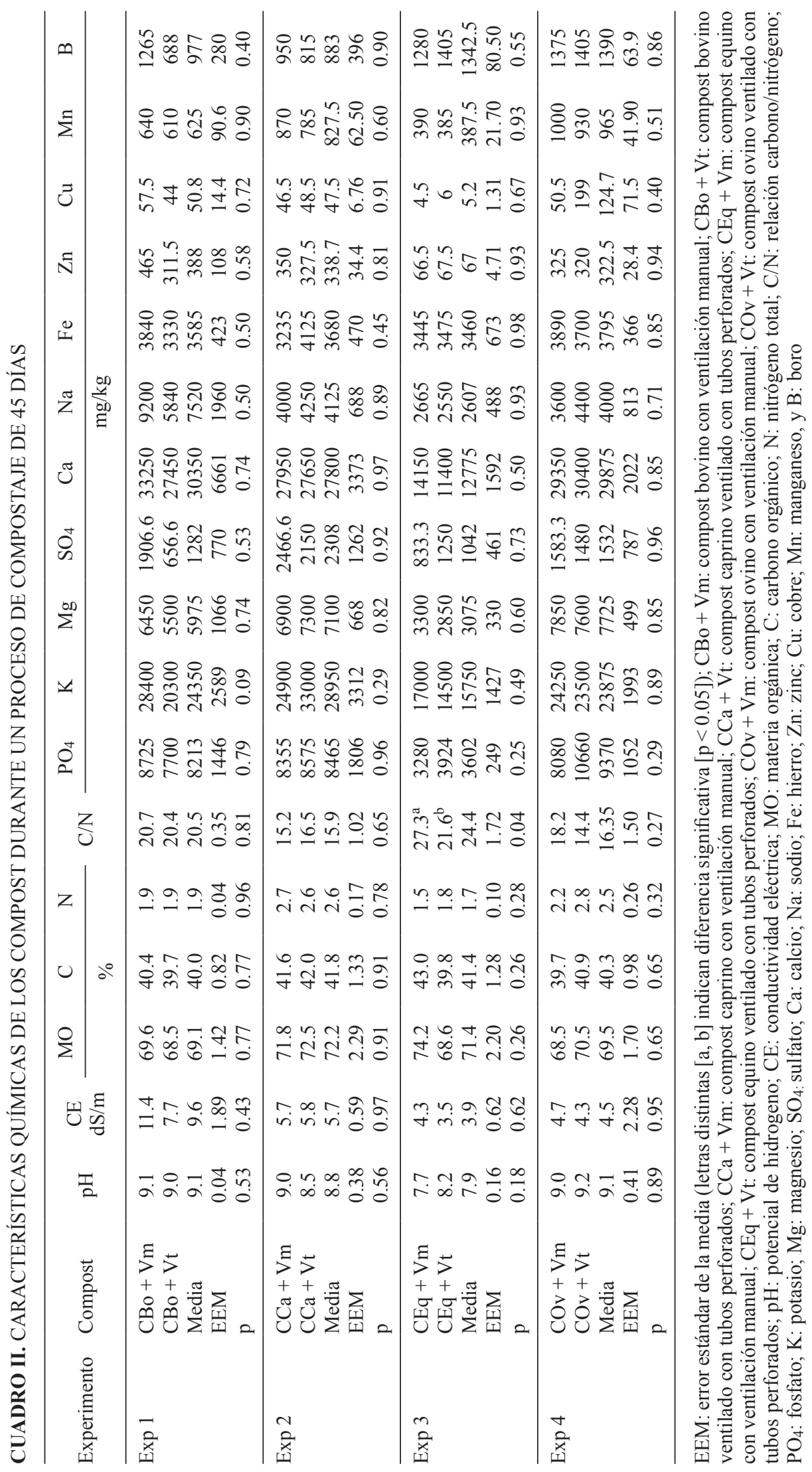


de compost con rangos de $\mathrm{CE}<12 \mathrm{dS} / \mathrm{m}$ en paisajes, áreas verdes urbanas y reforestación. En $\mathrm{CBo}+\mathrm{Vm}$ y $\mathrm{CBo}+\mathrm{Vt}$ disminuyó el contenido de $\mathrm{N}$, lo cual pudo deberse a perdidas por lixiviación debido al exceso de humedad que contenían los productos ( $>80 \%$ ) al inicio del proceso. El contenido de $\mathrm{K}, \mathrm{Mg}, \mathrm{Ca}, \mathrm{Na}$ y Zn se relacionó con ingesta de sales minerales contenidos en la suplementación que recibió el ganado.

Exp 2. El CCa no mostró diferencias estadísticas $(p>0.05)$ en la composición química por efecto de los métodos de ventilación. Sin embargo, se observó que el contenido de $\mathrm{N}$ total del producto aumentó respecto al contenido inicial del estiércol. Esto ocurre cuando las bacterias nitrificadoras oxidan inmediatamente el amonio liberado por la mineralización de los compuestos orgánicos nitrogenados y éste no se pierde por volatilización (Insam y de Bertoldi 2007). Brito et al. (2008) mencionan que el aumento de $\mathrm{N}$ se debe a la adecuada relación $\mathrm{C} / \mathrm{N}$ del material inicial, la cual es eficiente para la retención del $\mathrm{N}$ liberado por la degradación microbiana y para disminuir el riesgo por volatilización de amoniaco $\left(\mathrm{NH}_{3}\right)$. La relación $\mathrm{C} / \mathrm{N}$ inicial en el estiércol fue de 22.2 y al final del proceso fue de 15.2 en $\mathrm{CCa}+\mathrm{Vm}$ y de 16.5 en $\mathrm{CCa}$ $+\mathrm{Vt}$. Estos valores concuerdan con lo reportado por Bueno et al. (2008) en compost de residuos de poda de Leucaena (C/N: 12.6-21.5). Los valores de C/N colocan al compost caprino como mejorador de suelo con uso en agricultura ecológica y reforestación según la NADF-020-AMBT-2011 (SEDEMA 2012).

Exp 3. El CEq mostró diferencias estadísticas ( $\mathrm{p}$ $=0.049$ ) en la variable $\mathrm{C} / \mathrm{N}$. CEq $+\mathrm{Vt}$ presentó menor valor en la relación $\mathrm{C} / \mathrm{N}$ respecto a $\mathrm{CEq}+\mathrm{Vm}$, lo cual podría indicar que en este producto el método de ventilación con tubos perforados favoreció la mayor degradación de la materia orgánica a pesar de que promovió la disminución en la retención de calor en las pilas. Guo et al. (2012) indicaron que la tasa de aireación fue el factor que tuvo mayor influencia en la estabilidad del compost, en tanto que la $\mathrm{C} / \mathrm{N}$ inicial influyó principalmente en la maduración del compostaje con estiércol porcino. Said y Gigliotti (2007) y Bernal et al. (2009) comentaron que durante la fase activa del proceso de compostaje el carbono orgánico disminuye en el material debido a la descomposición de MO por los microorganismos, y esta disminución de $\mathrm{MO}$ reduce la $\mathrm{C} / \mathrm{N}$. El $\mathrm{pH}$ en CEq fue en promedio 7.9 y la CE 3.9. La CE refleja la salinidad de una enmienda orgánica. El alto contenido de sales puede causar problemas de fitotoxicidad, y por lo tanto es un buen indicador para un uso conveniente y seguro del compost en la agricultura. La norma NTEA-006-SMA-RS-2006
(SMA 2006) indica que un compost debe cumplir con las características de $\mathrm{pH} 6.5$ a $8, \mathrm{MO}>15 \%$ , $\mathrm{C} / \mathrm{N} \leq 12, \mathrm{P}=1 \mathrm{~g} / \mathrm{kg}, \mathrm{K}=2.5 \mathrm{~g} / \mathrm{kg}$, relación $\mathrm{K} /$ $\mathrm{Na}>2.5, \mathrm{Cu}=0.03 \mathrm{~g} / \mathrm{kg}$ y $\mathrm{Zn}=0.09 \mathrm{~g} / \mathrm{kg}$; en este trabajo, el CEq cumplió con dichas características excepto en la relación $\mathrm{C} / \mathrm{N}$.

Exp 4. El producto ovino no presentó diferencias estadísticas $(p>0.05)$ en la composición química por efecto de los tratamientos. El valor del $\mathrm{pH}$ en $\mathrm{COv}+$ $\mathrm{Vm}$ fue de 9 y en $\mathrm{COv}+\mathrm{Vt}$ 9.2, mientras que la $\mathrm{CE}$ fue 4.7 y $4.3 \mathrm{dS} / \mathrm{m}$, respectivamente. Gómez-Brandón et al. (2008) reportaron $\mathrm{pH}$ mayores a 9 después de 80 días de compostaje de estiércol bovino, supuestamente debido a humectación del compost maduro con lixiviados obtenidos de los establos y al volteo de las pilas. Cook et al. (2015) reportaron un incremento esperado en $\mathrm{Al}, \mathrm{Ca}, \mathrm{Fe}, \mathrm{K}$ y $\mathrm{S}$ debido a la limitada movilidad de los compuestos y la concentración elemental, debido a la evolución del $\mathrm{CO}_{2}$ y el incremento de la materia seca. También reportaron disminución en la concentración de N, P, Mg y Zn en tratamientos de compostaje de una mezcla de purines de cerdo con aserrín durante dos estaciones del año (otoño y verano). De acuerdo con la concentración máxima permitida de elementos traza $(150 \mathrm{mg} / \mathrm{kg}$ de $\mathrm{Cu}$ y $500 \mathrm{mg} / \mathrm{kg}$ de $\mathrm{Zn}$ ) en la norma NADF-020-AMBT-2011 (SEDEMA 2012) el COv se clasifica en nivel 2 tipo $\mathrm{B}$ con uso en agricultura ecológica y reforestación.

\section{CONCLUSIONES}

Los $\mathrm{CBo}, \mathrm{CCa}, \mathrm{y} \mathrm{COv}$ no mostraron variación en las características químicas por efecto del método de ventilación. En el CEq el método de ventilación con tubos perforados de policloruro de vinilo favoreció la reducción de la relación $\mathrm{C} / \mathrm{N}$, indicando que el espacio de aire libre en los tubos es funcional para la degradación de la materia orgánica, ya que fomenta mayor mineralización de los compuestos; sin embargo, cuando el estiércol equino se encuentre mezclado con paja de avena, la relación $\mathrm{C} / \mathrm{N}$ deberá balancearse adecuadamente en la mezcla inicial del sustrato adicionando desechos nitrogenados, lo cual dependerá de los contenidos iniciales del estiércol y de la paja de avena.

\section{AGRADECIMIENTOS}

A la Secretaría de Investigación y Estudios Avanzados de la Universidad Autónoma del Estado 
de México, por el financiamiento del proyecto $3745 / 2014 /$ CIA, cuyos resultados se utilizaron para elaborar este artículo.

\section{REFERENCIAS}

Azzez J.O. y Van Averbeke W. (2010). Nitrogen mineralization potential of three animal manures applied on a sandy clay loam soil. Bioresour. Technol. 101 (14), 5645-5651. DOI: 10.1016/j.biortech.2010.01.119

Bernal M.P., Alburquerque J.A. y Moral R. (2009). Composting of animal manures and chemical criteria for compost maturity assessment. A review. Bioresour. Technol. 100 (22), 5444-5453.

DOI: $10.1016 /$ j.biortech.2008.11.027

Brito L.M., Coutinho J. y Smith S.R. (2008). Methods to improve the composting process of the solid fraction of dairy cattle slurry. Bioresour. Technol. 99 (18), 89958960. DOI: 10.1016/j.biortech.2008.05.005

Bueno P., Tapias R., López F. y Díaz M.J. (2008). Optimizing composting parameters for nitrogen conservation in composting. Bioresour. Technol. 99 (11), 5069-5077. DOI: $10.1016 /$ j.biortech.2007.08.087

Cáceres R., Flotats X. y Marfà O. (2006). Changes in the chemical and physicochemical properties of the solid fraction of cattle slurry during composting using different aeration strategies. Waste Manage. 26 (10), 1081-1091. DOI: 10.1016/j.wasman.2005.06.013

Chadwick D.R., John F., Pain B.F., Chambers B.J. y Williams J.C. (2000). Plant uptake of nitrogen form the organic nitrogen fraction of animal manures: A laboratory experiment. J. Agric. Sci. Camb. 134 (2), 159-168.

Cook K.L, Ritchey E.L., Loughrin J.H., Haley M., Sistani K.R. y Bolster C.H. (2015). Effect of turning frequency and season on composting materials from swine high-rise facilities. Waste Manage. 39, 86-95. DOI: 10.1016/j.wasman.2015.02.019

Diaz L.F. y Savage G.M. (2007). Factors that affect the process. En: Compost science and technology (L.F. Diaz, M. de Bertoldi, W. Bidlingmaier y E. Stentiford, Eds.). Waste Management Series 8. Elsevier, Amsterdam, 357 pp.

El Kader N.A., Robin P., Paillat J.M. y Leterme P. (2007). Turning, compacting and the addition of water as factors affecting gaseous emissions in farm manure composting. Bioresour. Technol. 98 (14), 2619-2628. DOI: 10.1016/j.biortech.2006.07.035

Gómez-Brandón M., Lazcano C. y Domínguez J. (2008). The evaluation of stability and maturity during the composting of cattle manure. Chemosphere 70 (3), 436-444. DOI: 10.1016/j.chemosphere.2007.06.065

Guo R., Li G., Jiang T., Schuchardt F., Chen T., Zhao Y. y Shen Y. (2012). Effect of aeration rate, $\mathrm{C} / \mathrm{N}$ ratio and moisture content on the stability and maturity of compost. Bioresour. Technol. 112, 171-178.

DOI: 10.1016/j.biortech.2012.02.099

INEGI (2009). Prontuario de información geográfica municipal de los Estados Unidos Mexicanos. San Simón de Guerrero, México. Instituto Nacional de Estadística y Geografía, México, 9 pp. [en línea]. http://www3. inegi.org.mx/contenidos/app/mexicocifras/datos_geograficos/15/15077.pdf 20/01/2015

Insam H. y de Bertoldi M. (2007). Microbiology of the composting process. En: Compost science and technology (L.F. Diaz, M. de Bertoldi, W. Bidlingmaier y E. Stentiford, Eds.). Waste Management Series 8. Elsevier, Amsterdam, $357 \mathrm{pp}$.

Kulcu R. y Yaldiz O. (2007). Composting of goat manure and wheat straw using pine cones as a bulking agent. Bioresour. Technol. 98 (14), 2700-2704.

DOI: 10.1016/j.biortech.2006.09.025

Lazcano C., Gómez-Brandón M. y Domínguez J. (2008). Comparison of the effectiveness of composting and vermicomposting for the biological stabilization of cattle manure. Chemosphere 72 (7), 1013-1019.

DOI: 10.1016/j.chemosphere.2008.04.016

Li L.-1. y Li S-t. (2014). Nitrogen mineralization from animal manures and its relation to organic $\mathrm{N}$ fraction. J. Integr. Agric. 13 (9), 2040-2048. DOI: 10.1016/S2095-3119(14)60769-3

Luo W.H., Yuan J., Luo Y.M., Li G.X., Nghiem L.D. y Price W.E. (2014). Effects of mixing and covering with mature compost on gaseous emissions during composting. Chemosphere 117, 14-19.

DOI: 10.1016/j.chemosphere.2014.05.043

Mirabelli E. (2008). El compostaje proyectado a la lombricultura. Editorial Hemisferio Sur, Buenos Aires, 324 pp.

Oviedo-Ocana E.R., Marmolejo-Rebellón L.F. y TorresLozada P. (2014). Influencia de la frecuencia de volteo para el control de la humedad de los sustratos en el compostaje de biorresiduos de origen municipal. Rev. Int. Contam. Ambie. 30 (1), 91-100.

Parkinson R., Gibbs P., Burchett S. y Misselbrook T. (2004). Effect of turning regime and seasonal weather conditions on nitrogen and phosphorus losses during aerobic composting of cattle manure. Bioresour. Technol. 91 (2), 171-178. DOI: 10.1016/S0960-8524(03)00174-3

Pinos-Rodríguez J.M., García-López J.C., Peña-Avelino L.Y., Rendón-Huerta J.A., González- González C. y Tristán-Patiño F. (2012). Impactos y regulaciones ambientales del estiércol generado por los sistemas ganaderos de algunos países de América. Agrociencia 46 (4), 359-370.

Ruiz-Fernández J.F. (2012). Ingeniería del compostaje. Universidad Autónoma Chapingo, Texcoco, México, $237 \mathrm{pp}$. 
Said P.D. y Gigliotti G. (2007). Oxidative biodegradation of dissolved organic matter during composting. Chemosphere 68 (6), 1030-1040.

DOI: 10.1016/j.chemosphere.2007.02.012

SEDEMA (2012). Norma ambiental para el Distrito Federal NADF-020-AMBT-2011. Requerimientos mínimos para la producción de composta a partir de la fracción orgánica de los residuos sólidos urbanos, agrícolas, pecuarios y forestales, así como las especificaciones mínimas de calidad de la composta producida y/o distribuida en el Distrito Federal. Secretaría del Medio Ambiente del Gobierno del Distrito Federal. Gaceta Oficial del Distrito Federal. 30 de noviembre.
SEECO (2008). Norma mexicana NMX-FF-109-SCFI-2008. Humus de lombriz (lombricomposta). Especificaciones y métodos de prueba. Secretaría de Economía. Diario Oficial de la Federación, 10 de junio. SMA (2006). Norma técnica estatal ambiental NTEA-006SMA-RS-2006. Requisitos para la producción de los mejoradores de suelos elaborados a partir de residuos orgánicos. Secretaría de Medio Ambiente del Estado de México, Gaceta del Gobierno, 9 de octubre.

Szabová E., Juriš P. y Papajová I. (2010). Sanitation composting process in different seasons. Ascaris suum as model. Waste Manage. 30 (3), 426-432.

DOI: 10.1016/j.wasman.2009.09.035 